\title{
Ethnic Differences in Prevalence of Peripheral Artery Disease in Patients Undergoing Hemodialysis
}

\author{
Meghan Sebastianski • Marcello Tonelli • Ross T. Tsuyuki
}

Received: 12 August 2014 / Revised: 14 October 2014 / Accepted: 24 October 2014 / Published online: 16 December 2014

(C) W. Montague Cobb-NMA Health Institute 2014

\begin{abstract}
Background Hemodialysis patients experience poor outcomes associated with the presence of atherosclerosis, particularly lower-extremity peripheral artery disease (PAD). Prevalence of PAD is known to vary between ethnic groups; however, no information on ethnic-specific PAD prevalence in a hemodialysis cohort is available.

Methods Data from the Canadian Kidney Dialysis Cohort Study was used in a secondary analysis of 1293 adults starting hemodialysis in three major Canadian centres. PAD diagnosis was determined through structured interview and supplemented by clinical record. Ethnicity was self-reported.

Results Overall PAD prevalence was $19.1 \%$ with no significant difference between ethnic groups. Ethnic differences observed in diabetes prevalence in the full hemodialysis group were not present in the subset of PAD patients.

Conclusions The prevalence of PAD in patients undergoing hemodialysis is high, however we found no apparent ethnic differences in prevalence between ethnic groups.
\end{abstract}

Keywords Peripheral artery disease $\cdot$ Chronic kidney disease $\cdot$ Hemodialysis $\cdot$ Ethnic groups

M. Sebastianski $\cdot$ R. T. Tsuyuki $(\bowtie)$

Epidemiology Coordinating and Research (EPICORE) Centre, Suite 4000, Research Transition Facility (RTF), University of Alberta, 8308-144 Street NW, Edmonton, Alberta T6G 2V2, Canada e-mail: ross.tsuyuki@ualberta.ca

M. Tonelli

Division of Nephrology, Department of Medicine, University of Alberta, Edmonton, Alberta, Canada

R. T. Tsuyuki

Division of Cardiology, Department of Medicine, University of Alberta, Edmonton, Alberta, Canada

\section{Introduction}

Patients with chronic kidney disease (CKD) requiring hemodialysis have high rates of lower-extremity atherosclerosis, where prevalence of peripheral artery disease (PAD) ranges from 16.6 to $38.5 \%$, compared to 4.4 to $29 \%$ in the general population [1-4]. In patients receiving hemodialysis, PAD is associated with increased hospitalization (hazard ratio (HR) $1.19, p<0.0001)$ and increased rates of myocardial infarction (MI) and stroke [5, 6]. Concomitant PAD is also an independent predictor of mortality in hemodialysis patients (HR 1.67, $p=0.004)$ [7].

Similarly, PAD is also an independent predictor of these poor outcomes in patients with coronary artery disease (CAD), and within this population, there is a significant ethnic variation in PAD prevalence [8-10]. Black populations have high rates of diabetes and $C A D$ and a high prevalence of $P A D$ compared to all other ethnic groups [11-13]. While South Asians also have a high prevalence of PAD risk factors including diabetes and CAD, they appear to have a paradoxically lower prevalence of PAD compared to White Europeans [14].

Prognosis of CKD also varies among ethnic groups [15]. Chinese and South Asians at equal glomerular filtration rates (GFR) and proteinuria levels have a lower risk of mortality than Whites [15]. When comparing Black and White ethnic groups, although the Black population develops end stage renal disease (ESRD) much earlier, once the disease progresses to where the patient requires dialysis, mortality in the White population becomes significantly higher [10].

While ethnic differences have been observed in both CKD and PAD prevalence, few studies have focused on the ethnic PAD prevalence within a hemodialysis cohort [10]. Our study examines the differences in PAD prevalence in patients undergoing hemodialysis. 


\section{Methods}

\section{Study Design}

We conducted a secondary analysis of data from the Canadian Kidney Dialysis Cohort Study (CKDCS) [16]. The CKDCS is a large, prospective observational study of all consenting adults commencing hemodialysis treatment in multiple ethnically diverse major centres across Canada [16]. CKDCS was conducted according to the principles of the Declaration of Helsinki and approved by the ethics boards of each participating centre [16].

\section{Study Participants}

Our study sample included 1293 adult $(\geq 18$ years of age) hemodialysis patients from Edmonton, Calgary and Vancouver. All available patients from the database were included provided they had completed the baseline cohort study questionnaire between March 23, 2005, and December 20, 2011.

\section{Data Collection}

Baseline demographic data and clinical history from CKDCS were collected by structured interview and supplemented by clinical record. Ethnicity was self-identified. The baseline data collection was conducted within 8 weeks of the patient starting hemodialysis.

\section{Data Analysis}

Ethnicities were separated into groups based on the following classifications used by the CKDCS: Aboriginal (North American Indian, Métis, Inuit); Asian (Chinese, Japanese, Vietnamese, Korean, Taiwanese); Black (African, Caribbean, South American, Cuban); Middle Eastern (Saudi Arabian, Iranian, Iraqi, Algerian, Jordanian, Syrian, Armenian); Pacific Islander (Filipino, Fijian, Hawaiian, Samoan); South Asian (Indian, Pakistani, Bangladeshi) and White/Caucasian (French Canadian, European, Australian, Russian ancestry or non-black Hispanic). For this secondary analysis, the patient was considered to have PAD if they answered yes to having any one of the following: previous claudication symptoms, current claudication symptoms, gangrene, previous vascular intervention or previous amputation due to vascular disease. Data are presented as number of cases with characteristic of interest and as a percent of the total available responses for that ethnic group. Prevalence differences were compared using Chi-squared tests and oneway ANOVA with Tukey's HSD (SPSS Inc., Version 17: Chicago, IL, USA).

\section{Results}

Characteristics of the patients included in the study, stratified by ethnic group, are shown in Table 1 . The mean age of the population sample was 60.3 years (standard deviation (SD) 15.6). Aboriginals were significantly younger compared to Whites $(p<0.001)$. Prevalence of hypertension ranged from $84.6 \%$ in Middle Eastern patients to $94.8 \%$ in South Asians. Documented CAD was less common, with an average prevalence of $24.7 \%$ ranging from $7.7 \%$ in Middle Easterners to $25.8 \%$ in Aboriginals. Diabetes was present in $51.4 \%$ of the cohort, with Aboriginals having a significantly higher prevalence compared to Whites ( 68.8 vs. $48.3 \%, p<0.001)$. Compared to Aboriginals, Blacks and Whites, South Asians had borderline significantly fewer cases of long-duration diabetes $(>10$ years, $p=0.046$ ). Large diversity in smoking habits were found across the ethnic groups with Whites and Aboriginals having significantly lower rates of non-smokers compared to the other ethnic groups.

In the 247 patients identified with symptomatic PAD, there were no significant ethnic differences in the comorbidities between ethnic groups with the exception of patients who had never smoked, warfarin use, and patients diagnosed with diabetes within the last year (Table 2). Asians and South Asians had significantly less smokers compared to Whites, while Whites had significantly less warfarin use compared to Blacks and South Asians, albeit with very few cases. The mean age was 60.8 years (SD 13.7) and the majority of patients were male (59.5\%). Hypertension was highly prevalent with an average of $90.7 \%$, ranging from $89.2 \%$ in Whites to $100 \%$ in five of the seven ethnic groups. The overall prevalence of diabetes was also high at $66.8 \%$, ranging from $65.5 \%$ in Whites to $100 \%$ in Pacific Islanders.

Overall PAD prevalence was $19.1 \%(n=247)$, ranging from 7.7 to $20.3 \%$ across the various ethnic groups. PAD prevalence was highest in Whites $(20.3 \%)$ followed by Aboriginals $(19.4 \%)$. The lowest prevalence was in persons from the Middle East (7.7 \%) followed by Pacific Islanders (9.8\%). We found no statistically significant differences in PAD prevalence between ethnic groups.

\section{Discussion}

PAD has been associated with higher morbidity and mortality in both CKD and CAD patients $[5,10]$. Ethnic variations in prevalence of PAD have been found in the CAD population, yet little data is available to examine these patterns in patients with CKD receiving hemodialysis [10]. In our study, we found a high prevalence of symptomatic PAD, but did not find any statistically significant differences in PAD prevalence by ethnicity, suggesting ethnicity may not play a major role in PAD prevalence in this population. 


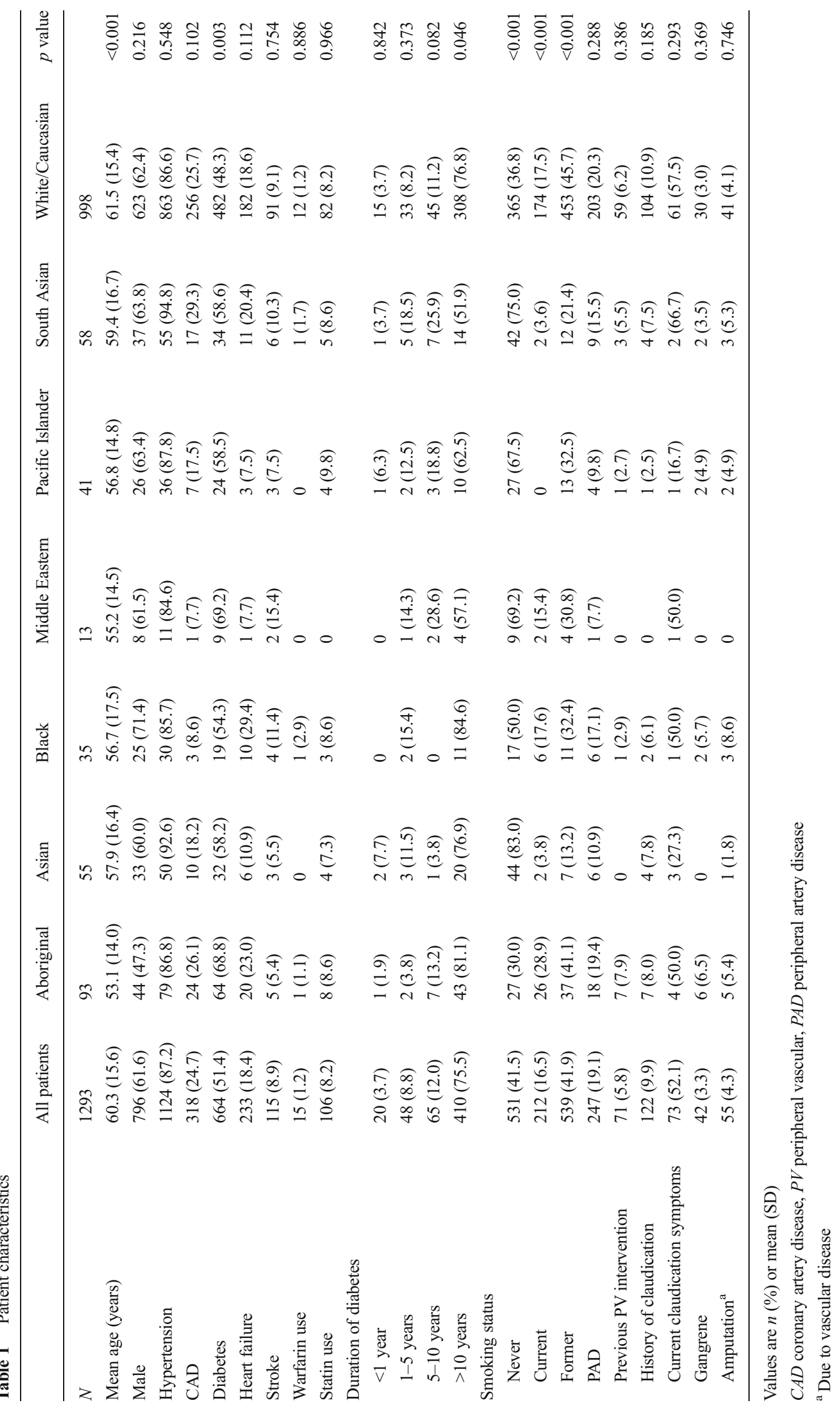


Table 2 Characteristics of patients with PAD

\begin{tabular}{|c|c|c|c|c|c|c|c|c|c|}
\hline & All patients & Aboriginal & Asian & Black & $\begin{array}{l}\text { Middle } \\
\text { Eastern }\end{array}$ & Pacific Islander & South Asian & White/Caucasian & $p$ value \\
\hline$N$ & 247 & 18 & 6 & 6 & 1 & 4 & 9 & 203 & \\
\hline Mean age (years) & 60.8 (13.7) & $54.1(9.9)$ & $54.9(13.9)$ & $57.3(20.9)$ & 69.4 & $63.7(9.2)$ & $63.2(14.2)$ & $63.1(13.6)$ & 0.119 \\
\hline Male & 147 (59.5) & $10(55.6)$ & $4(66.7)$ & $4(66.7)$ & $1(100)$ & $4(100)$ & $5(55.6)$ & $119(58.6)$ & 0.690 \\
\hline Hypertension & 224 (90.7) & $17(94.4)$ & $6(100)$ & $6(100)$ & $1(100)$ & $4(100)$ & $9(100)$ & $181(89.2)$ & 0.740 \\
\hline CAD & $90(36.4)$ & $5(27.8)$ & $1(16.7)$ & $2(33.3)$ & 0 & $3(75.0)$ & $1(11.1)$ & $80(39.4)$ & 0.076 \\
\hline Diabetes & $165(66.8)$ & $12(66.7)$ & $5(83.3)$ & $5(83.3)$ & 0 & $4(100.0)$ & $6(66.7)$ & $133(65.5)$ & 0.466 \\
\hline Heart failure & $64(26.6)$ & $4(25.0)$ & 0 & $2(33.3)$ & $1(100)$ & $1(25.0)$ & $2(25.0)$ & $54(27.0)$ & 0.527 \\
\hline Stroke & $26(10.5)$ & $1(5.6)$ & 0 & 0 & 0 & 0 & 0 & $25(12.3)$ & 0.647 \\
\hline Warfarin use & $3(1.2)$ & 0 & 0 & $1(16.7)$ & 0 & 0 & $1(11.1)$ & $1(0.5)$ & 0.002 \\
\hline Statin use & $24(9.7)$ & 0 & 0 & $1(16.7)$ & 0 & 0 & $3(33.3)$ & $20(9.9)$ & 0.164 \\
\hline \multicolumn{10}{|c|}{ Duration of diabetes } \\
\hline$<1$ year & $2(1.4)$ & 0 & $1(20.0)$ & 0 & 0 & 0 & 0 & $1(0.8)$ & 0.021 \\
\hline 1 to 5 years & $9(6.2)$ & 0 & 0 & 0 & 0 & 0 & 0 & $9(7.6)$ & 0.836 \\
\hline 5 to 10 years & $17(11.7)$ & $1(8.3)$ & 0 & 0 & 0 & $1(50.0)$ & $2(50.0)$ & $13(10.9)$ & 0.082 \\
\hline$>10$ years & $117(80.7)$ & $11(91.7)$ & $5(83.3)$ & $3(100)$ & 0 & $1(50.0)$ & $2(50.0)$ & $96(80.7)$ & 0.383 \\
\hline \multicolumn{10}{|l|}{ Smoking status } \\
\hline Never & $76(31.1)$ & $4(22.2)$ & $5(100)$ & $1(16.7)$ & 0 & $2(50.0)$ & $6(66.7)$ & $58(28.9)$ & 0.004 \\
\hline Current & $50(20.5)$ & $5(27.8)$ & 0 & $2(33.3)$ & 0 & 0 & 0 & $43(21.4)$ & 0.402 \\
\hline Former & $118(47.8)$ & $9(50.0)$ & 0 & $3(50.0)$ & $1(100)$ & $2(50.0)$ & $3(33.3)$ & $54(27.0)$ & 0.345 \\
\hline
\end{tabular}

Values are $n(\%)$ or mean (SD)

$C A D$ coronary artery disease, $P V$ peripheral vascular, $P A D$ peripheral artery disease

Overall, we found a PAD prevalence of $19.1 \%$ in the hemodialysis cohort. These results agree with previous studies which found a $19 \%$ prevalence in European patients with chronic renal failure stages IV/V [17] (no dialysis) and a study of ESRD hemodialysis patients across ten European countries where the PAD prevalence ranged from 17.5 to $37.8 \%$ [5]. These rates are slightly higher than the $15 \%$ reported by the United States Renal Data System (USRDS) of patients undergoing incident dialysis where PAD was defined using only clinical symptoms [2]. Although we found the prevalence among Asians was numerically lower than in people of other ethnicities at $10.9 \%$, the difference was not statistically significant. However, studies done in Japan have reported a PAD prevalence of $22.1 \%$ in hemodialysis patients using the gold standard ankle-brachial index (ABI) diagnosis [7], which reflects a limitation in our case definition (as up to $50 \%$ of patients with PAD are asymptomatic) [4].

One unexpected finding was a similar PAD prevalence between Whites and South Asians. Previous research has indicated that South Asians have significantly less PAD compared to White Europeans [14]. We hypothesized this ethnic disparity would be similar in hemodialysis patients. One possible explanation for the similar rates is the select nature of hemodialysis populations. Specifically, since vascular disease may contribute to both CKD and PAD, it is possible that the presence of CKD selects for people who are at excess risk of
PAD — regardless of ethnicity. Similar considerations might apply to other factors (such as vasculitis) which could contribute to the risk of both CKD and PAD. Further research into changes of PAD prevalence through different stages of CKD for the different ethnic groups may shed some light on this finding.

This study was limited in that the gold standard for diagnosis of PAD (ABI) was not available. Sole reliance on clinical symptoms likely results in an up to $85 \%$ underestimation of PAD prevalence [4]. There may also be differences in symptom reporting between ethnic groups. In addition, the number of cases in each ethnic group with PAD was relatively small; therefore, we may have been statistically underpowered to detect a difference.

\section{Conclusion}

We did not observe ethnic differences in PAD prevalence in this hemodialysis cohort. Ethnicity is not likely a key factor in the prevalence of PAD in hemodialysis patients. The high prevalence of PAD supports further research in the utility of PAD screening in early stages of CKD to implement treatments for PAD earlier. 
Acknowledgments The authors would like to thank Natasha Wiebe for her contributions in utilizing the Canadian Kidney Dialysis Cohort Study database.

Conflict of Interest M. Sebastianski, M. Tonelli and RT Tsuyuki declare that they have no conflict of interest.

Funding This work was supported by the Canadian Institutes for Health Research (MOP 133582) and a Leaders Opportunity Fund grant from the Canada Foundation for Innovation.

Informed Consent All procedures followed were in accordance with the ethical standards of the responsible committee on human experimentation (institutional and national) and with the Helsinki Declaration of 1975 , as revised in 2000. Informed consent was obtained from all patients for being included in the study.

\section{References}

1. O’Hare A, Johansen K. Lower-extremity peripheral arterial disease among patients with end-stage renal disease. J Am Soc Nephrol. 2001;12:2838-47.

2. Saha HHT, Leskinen YKJ, Salenius JP, Lahtela JT. Peripheral vascular disease in diabetic peritoneal dialysis patients. Perit Dial Int. 2007;27:S210-4.

3. Makowsky M, McMurtry MS, Elton T, Rosenthal M, Gunther M, Percy M, et al. Prevalence and treatment patterns of lower extremity peripheral arterial disease among patients at risk in ambulatory health settings. Can J Cardiol. 2011;27(3):389.e11-e18.

4. Hirsch AT, Criqui MH, Treat-Jacobson D, Regensteiner JG, Creager MA, Olin JW, et al. Peripheral arterial disease detection, awareness, and treatment in primary care. JAMA. 2001;286(11):1317-24.

5. Rajagopalan S, Dellegrottaglie S, Furniss AL, Gillespie BW, Satayathum S, Lameire N, et al. Peripheral arterial disease in patients with end-stage renal disease: observations from the dialysis outcomes and practice patterns study (DOPPS). Circulation. 2006;114:1914-22.

6. Paraskevas KI, Koupidis SA, Tzovaras AA, Nikolaou A, Mikhailidis DP. Screening for peripheral artery disease in dialysis patients: an opportunity of early disease detection and timely initiation of appropriate therapeutic measures. Int Urol Nephrol. 2011;43:143-5.
7. Otsubo S, Kitamura M, Wakaume T, Yajima A, Ishihara M, Takasaki $\mathrm{M}$, et al. Association of peripheral artery disease and long-term mortality in hemodialysis patients. Int Urol Nephrol. 2012;44:569-73.

8. Abramson BL, Huckell V, Anand S, Forbes T, Gupta A, Harris K, et al. Canadian cardiovascular society consensus conference: peripheral arterial disease - executive summary. Can J Cardiol. 2005;21:997-1006.

9. Hirsch AT, Haskal ZJ, Hertzer NR, Bakal CW, Creager MA, Haperin $\mathrm{JL}$, et al. ACC/AHA 2005 guidelines for the management of patients with peripheral arterial disease (lower extremity, renal, mesenteric, and abdominal aortic): a collaborative report from the American Association for Vascular Surgery/Society for Vascular Surgery, Society for Cardiovascular Angiography and Interventions, Society for Vascular Medicine and Biology, Society for Interventional Radiology, and the ACC/AHA Task Force on Practice Guidelines (Writing Committee to Develop Guidelines for the Management of Patients with Peripheral Arterial Disease). J Am Coll Cardiol. 2006;47:1239-312.

10. Bennett PC, Silverman S, Gill PS, Lip GY. Ethnicity and peripheral artery disease. QJM. 2009;102(1):3-16.

11. Nelson KM, Reiber G, Kohler T, Boyko EJ. Peripheral arterial disease in a multiethnic national sample: the role of conventional risk factors and allostatic load. Ethn Dis. 2007;17:669-75.

12. Allison MA, Peralta CA, Wassel CL, Aboyans V, Arnett DK, Cushman M, et al. Genetic ancestry and lower extremity peripheral artery disease in the multi-ethnic study of atherosclerosis. Vasc Med. 2010;15:351-9.

13. Lloyd-Jones D, Adams RJ, Brown TM, Carnethon M, Dai S, De Simone G, et al. Heart disease and stroke statistics - 2010 update: a report from the American Heart Association. Circulation. 2010;121: e1-e170.

14. Sebastianski M, Makowsky MJ, Dorgan M, Tsuyuki RT. Paradoxically lower prevalence of peripheral arterial disease in South Asians: a systematic review and meta-analysis. Heart. 2013. doi:10.1136/heartjnl-2013-303605.

15. Conley J, Tonelli M, Quan H, Manns BJ, Palacios-Derflingher L, Bresee LC, et al. Association between GFR, proteinuria, and adverse outcomes among White, Chinese, and South Asian individuals in Canada. Am J Kidney Dis. 2012;59(3):390-9.

16. Bello AK, Thadhani R, Hemmelgarn B, Klarenbach S, Gill J, Chan $\mathrm{C}$, et al. Design and implementation of the Canadian Kidney Disease Cohort Study (CKDCS): a prospective observational study of incident hemodialysis patients. BMC Nephrol. 2011;12:10-9.

17. Guerrero A, Montes R, Munoz-Terol J, Gil-Peralta A, Toro J, Naranjo $\mathrm{M}$, et al. Peripheral arterial disease in patients with stages IV and V chronic renal failure. Nephrol Dial Transplant. 2006;21:3525-31. 